(n)

\title{
The Virasoro action on the tau function for the constrained discrete KP hierarchy
}

Jipeng Cheng, Maohua Li, Jingsong He

To cite this article: Jipeng Cheng, Maohua Li, Jingsong He (2013) The Virasoro action on the tau function for the constrained discrete KP hierarchy, Journal of Nonlinear Mathematical Physics 20:4, 529-538, DOI:

https://doi.org/10.1080/14029251.2013.868266

To link to this article: https://doi.org/10.1080/14029251.2013.868266

Published online: 04 January 2021 


\title{
The Virasoro action on the tau function for the constrained discrete KP hierarchy
}

\author{
Jipeng Cheng \\ Department of Mathematics, China University of Mining and Technology, \\ Xuzhou, Jiangsu 221116, P. R. China \\ Maohua Li and Jingsong $\mathrm{He}^{*}$ \\ Department of Mathematics, Ningbo University, \\ Ningbo, Zhejiang 315211, P. R. China \\ *hejingsong@nbu.edu.cn
}

Received 18 August 2013

Accepted 25 September 2013

\begin{abstract}
With the help of the squared eigenfunction potential, the action of the Virasoro symmetry on the tau function of the constrained discrete KP hierarchy is derived.

Keywords: the constrained discrete KP hierarchy; squared eigenfunction symmetry; additional symmetries.

2010 Mathematics Subject Classification: 35Q51, 37K10, 37K40
\end{abstract}

\section{Introduction}

The discrete Kadomtsev-Petviashvili (dKP) hierarchy [11,13] is an important research object in the field of the discrete integrable system, which is defined by the difference operator $\Delta$ (see Section 2) instead of $\partial_{x}$ in the usual KP hierarchy [8]. Recently, many researches have been done in this field. For example, 1) the additional symmetry and the gauge transformation for the dKP hierarchy and its constrained case [17-20]; 2) the squared eigenfunction symmetry (or the "ghost" symmetry) [14]; 3 ) the extended dKP hierarchy and its solutions [22]; 4) Lie algebraic structure of the dKP hierarchy and the links between the dKP hierarchy and the usual KP hierarchy [10,23].

The additional symmetry $[1,4-7,9,12,15,16,21,24-26]$ is a kind of symmetry depending explicitly on the space and time variables. It is involved in so-called string equation and the generalized Virasoro constraints in matrix models of the $2 \mathrm{~d}$ quantum gravity (see $[8,27]$ and references therein). The additional symmetry of the dKP hierarchy is investigated in [20]. However for the constrained dKP hierarchy, the conventional additional symmetry can not preserve the form of the Lax operator with additional constraint (see (3.1)) and some modification must be needed. In [18], Virasoro symmetry (one kind of the additional symmetries) for the constrained dKP hierarchy is constructed by adding the squared eigenfunction symmetry flows to the standard additional symmetry flows, and the corresponding algebraic structure is studied on the space of the Lax operator. But the action of the Virasoro symmetry on the tau function of the constrained dKP hierarchy has not been obtained.

${ }^{*}$ Corresponding author. 
The study of the Virasoro action on the tau function will be useful in establishing the link between the constrained dKP hierarchy and matrix models $[2,3]$. Similar to the usual constrained KP hierarchy [3], in this paper, we use the squared eigenfunction potential to investigate the action of the Virasoro symmetry on the tau function.

This paper is organized in the following way. In Section 2, we review some backgrounds on the dKP hierarchy. In Section 3, the Virasoro action on the tau function of the constrained dKP hierarchy is obtained. At last, some conclusions and discussions are given in Section 4.

\section{Backgrounds on the dKP hierarchy}

In this section, we will recall some basic facts about the dKP hierarchy. One can refer to $[11,14,20]$ for more details.

The dKP hierarchy can be expressed as

$$
\frac{\partial L}{\partial t_{i}}=\left[\left(L^{i}\right)_{+}, L\right], \quad i=1,2,3, \cdots
$$

where the Lax operator $L$ is given by a general first - order pseudo difference operator:

$$
L=L(n)=\Delta+\sum_{j=0}^{\infty} f_{j}(n) \Delta^{-j}
$$

Here $\Delta$ is the difference operator: $\Delta f(n)=f(n+1)-f(n)$, with the following rules for the multiplication of the function operators and the difference operators:

$$
\Delta^{j} f=\sum_{i=0}^{\infty} C_{j}^{i} \Delta(f)(n+j-i) \Delta^{j-i}, C_{j}^{i}=\frac{j(j-1) \cdots(j-i+1)}{i !},
$$

and $f_{j}(n)$ is the function of $n$ and the time variables $t=\left(t_{1}, t_{2}, t_{3}, \cdots\right)$. For the pseudo difference operator $R=R(n)=\sum_{j=-\infty}^{k} f_{j}(n) \Delta^{j}$ and the function $f=f(n), R_{+}=\sum_{j=0}^{k} f_{j}(n) \Delta^{j}, R_{-}=\sum_{j<0} f_{j}(n) \Delta^{j}$, $\operatorname{res}_{\Delta} R=f_{-1}(n)$, and $R(f)$ means the action of $R$ on $f$. The conjugation operation is defined in the following way: $(A B)^{*}=B^{*} A^{*}, \Delta^{*}=-\Delta \Lambda$ and $f^{*}=f$, where $A$ and $B$ are the pseduo difference operator, and $\Lambda$ is the shift operator: $\Lambda f(n)=f(n+1)$.

The dressing operator is defined as follows:

$$
W(n ; t)=1+\sum_{j=1}^{\infty} w_{j}(n ; t) \Delta^{-j}
$$

which satisfies

$$
L=W \Delta W^{-1} .
$$

Then the Lax equation(2.1) is equivalent to the Sato equation

$$
\frac{\partial W}{\partial t_{i}}=-\left(L^{i}\right)_{-} W
$$

The dKP hierarchy can be viewed as the compatibility conditions of the following linear problems:

$$
\begin{gathered}
L^{k}\left(\psi_{B A}(n ; t, \lambda)\right)=\lambda^{k} \psi_{B A}(n ; t, \lambda), \quad \partial_{t_{m}} \psi_{B A}(n ; t, \lambda)=L_{+}^{m}\left(\psi_{B A}(n ; t, \lambda)\right) \\
\text { Co-published by Atlantis Press and Taylor \& Francis } \\
\text { Copyright: the authors } \\
530
\end{gathered}
$$


and

$$
\left(L^{*}(n-1)\right)^{k}\left(\psi_{B A}^{*}(n ; t, \lambda)\right)=z^{k} \psi_{B A}^{*}(n ; t, \lambda), \quad \partial_{t_{m}} \psi_{B A}^{*}(n ; t, \lambda)=-\left(L^{*}(n-1)\right)_{+}^{m}\left(\psi_{B A}^{*}(n ; t, \lambda)\right),
$$

where $\psi_{B A}(n ; t, \lambda)$ is the Baker-Akhiezer (BA) wave function defined via:

$$
\begin{aligned}
\psi_{B A}(n ; t, \lambda) & =W(n ; t)\left((1+\lambda)^{n} e^{\xi(t, \lambda)}\right) \\
& =\left(1+\frac{w_{1}(n ; t)}{\lambda}+\frac{w_{2}(n ; t)}{\lambda^{2}}+\cdots\right)(1+\lambda)^{n} e^{\xi(t, \lambda)}
\end{aligned}
$$

and $\psi_{B A}^{*}(n ; t, \lambda)$ is the adjoint BA function:

$$
\begin{aligned}
\psi_{B A}^{*}(n ; t, \lambda) & =\left(W^{-1}(n-1 ; t)\right)^{*}\left((1+\lambda)^{-n} e^{-\xi(t, \lambda)}\right) \\
& =\left(1+\frac{w_{1}^{*}(n ; t)}{\lambda}+\frac{w_{2}^{*}(n ; t)}{\lambda^{2}}+\cdots\right)(1+\lambda)^{-n} e^{-\xi(t, \lambda)},
\end{aligned}
$$

where $\xi(t, \lambda)=\sum_{i=0}^{\infty} t_{i} \lambda^{i}$.

The dKP hierarchy can also be expressed in terms of a single function called the tau function $\tau_{\Delta}=\tau(n ; t)$, which is related with the wave function and the adjoint wave function in the following way,

$$
\begin{aligned}
& \psi_{B A}(n ; t, \lambda)=\frac{\tau\left(n ; t-\left[\lambda^{-1}\right]\right)}{\tau(n ; t)}(1+\lambda)^{n} e^{\xi(t, \lambda)}, \\
& \psi_{B A}^{*}(n ; t, \lambda)=\frac{\tau\left(n ; t+\left[\lambda^{-1}\right]\right)}{\tau(n ; t)}(1+\lambda)^{-n} e^{-\xi(t, \lambda)},
\end{aligned}
$$

where $[\lambda]=\left(\lambda, \frac{\lambda^{2}}{2}, \frac{\lambda^{3}}{3}, \cdots\right)$. There is a connection [11] between $\tau(t)$ of the usual KP hierarchy and $\tau(n ; t)$ of the dKP hierarchy, that is

$$
\tau_{\Delta}=\tau(n ; t)=\tau\left(t_{1}+n, t_{2}-\frac{n}{2}, t_{3}+\frac{n}{3}, \cdots\right) .
$$

If the functions $q(n ; t)$ and $r(n ; t)$ satisfy

$$
\partial_{t_{m}} q(n ; t)=L(n)_{+}^{m}(q(n ; t)), \quad \partial_{t_{m}} r(n ; t)=-\left(L(n)^{m}\right)_{+}^{*}(r(n ; t)),
$$

then they are called the eigenfunction and the adjoint eigenfunction of the dKP hierarchy respectively. Obviously, $\psi_{B A}(n ; t, \lambda)$ is the eigenfunction, while $\psi_{B A}^{*}(n+1 ; t, \lambda)$ is the adjoint eigenfunction. The relations between the (adjoint) eigenfunction and the (adjoint) wave function can be showed as the spectral representation below

$$
q(n ; t)=\int d \lambda \varphi(n ; \lambda) \psi_{B A}(n ; t, \lambda), \quad r(n ; t)=\int d \lambda \varphi^{*}(n ; \lambda) \psi_{B A}^{*}(n+1 ; t, \lambda),
$$

where two density functions $\varphi(n ; \lambda)$ and $\varphi^{*}(n ; \lambda)$ can be expressed by using the squared eigenfunction potentials

$$
\varphi(n ; \lambda)=-S\left(q\left(n ; t^{\prime}\right), \psi_{B A}^{*}\left(n+1 ; t^{\prime}, \lambda\right)\right), \quad \varphi^{*}(n ; \lambda)=S\left(\psi_{B A}\left(n ; t^{\prime}, \lambda\right), r\left(n ; t^{\prime}\right)\right) .
$$


Here for any pair of (adjoint) eigenfunctions $q(n ; t)$ and $r(n ; t)$, the squared eigenfunction potential $S(q(n ; t), r(n ; t))$ are determined by the following equations [14]:

$$
\begin{aligned}
& \Delta S(q(n ; t), r(n ; t))=q(n ; t) r(n ; t), \\
& \partial_{t_{m}} S(q(n ; t), r(n ; t))=\operatorname{res}_{\Delta}\left(\Delta^{-1} r(n ; t) L(n)_{+}^{m} q(n ; t) \Delta^{-1}\right)
\end{aligned}
$$

Note that the definition of $S(q(n ; t), r(n ; t))$ can be up to a constant. We listed some useful expressions of $S(q(n ; t), r(n ; t))$ which will be used later

$$
\begin{aligned}
& S\left(\psi_{B A}(n ; t, \mu), \psi_{B A}^{*}(n+1 ; t, \lambda)\right)=-\frac{1}{\lambda} \psi_{B A}\left(n ; t+\left[\lambda^{-1}\right], \mu\right) \psi_{B A}^{*}(n ; t, \lambda) \\
& =\frac{1}{\mu} \psi_{B A}(n ; t, \mu) \psi_{B A}^{*}\left(n ; t-\left[\mu^{-1}\right], \lambda\right)+\mathrm{constant}, \\
& S\left(q(n ; t), \psi_{B A}^{*}(n+1 ; t, \lambda)\right)=-\frac{1}{\lambda} q\left(n ; t+\left[\lambda^{-1}\right]\right) \psi_{B A}^{*}(n ; t, \lambda), \\
& S\left(\psi_{B A}(n ; t, \lambda), r(n ; t)\right)=\frac{1}{\lambda} \psi_{B A}(n ; t, \lambda) r\left(n-1 ; t-\left[\lambda^{-1}\right]\right), \\
& S(q(n ; t), r(n ; t))=\iint d \lambda d \mu \varphi^{*}(n ; \lambda) \varphi(n ; \mu) S\left(\psi_{B A}(n ; t, \mu), \psi_{B A}^{*}(n+1 ; t, \lambda)\right) .
\end{aligned}
$$

The squared eigenfunction symmetry for the dKP hierarchy, also called the "ghost" symmetry, is constructed in [14], which is defined by using pairs of the eigenfunctions and the adjoint eigenfunctions, i.e., $q_{i}(n ; t)$ and $r_{i}(n ; t), i=1,2, \cdots m$, that is,

$$
\partial_{\alpha} L=\left[\sum_{i=1}^{m} q_{i}(n ; t) \Delta^{-1} r_{i}(n ; t), L\right], \quad \partial_{\alpha} W=\left(\sum_{i=1}^{m} q_{i}(n ; t) \Delta^{-1} r_{i}(n ; t)\right) W,
$$

and its action on the tau function is

$$
\partial_{\alpha} \tau(n ; t)=-\sum_{i=1}^{m} S\left(q_{i}(n ; t), r_{i}(n ; t)\right) \tau(n ; t)
$$

The additional symmetry for the dKP hierarchy is defined by introducing the additional variables $\hat{t}_{m l}[20]$,

$$
\hat{\partial}_{m l} L=\left[-\left(M_{\Delta}^{m} L^{l}\right)_{-}, L\right], \quad \hat{\partial}_{m l} W=-\left(M_{\Delta}^{m} L^{l}\right)_{-} W,
$$

where

$$
M_{\Delta}=W \Gamma_{\Delta} W^{-1}, \quad \Gamma_{\Delta}=\sum_{i=1}^{\infty}\left(i t_{i} \Delta^{i-1}+(-1)^{i-1} n \Delta^{i-1}\right) .
$$

In particular, the additional symmetry $\hat{\partial}_{1, k}$ acts on the tau function in the way below [20]

$$
\hat{\partial}_{1, k} \tau(n ; t)=\frac{1}{2} \hat{W}_{k-1}^{(2)} \tau(n ; t)
$$

where

$$
\hat{W}_{k}^{(2)}=2 \sum_{i \geq 1}\left(i t_{i}+(-1)^{i-1} n\right) \frac{\partial}{\partial t_{i+k}}-(k+1) \frac{\partial}{\partial t_{k}}+\sum_{i=1}^{k-1} \frac{\partial^{2}}{\partial t_{i} \partial t_{k-i}} .
$$




\section{The Virasoro action on the tau function for the constrained dKP hierarchy}

In general, the constrained dKP hierarchy with $m$ components is defined by restricting the Lax operator in the following form of $L(n)_{-}=\sum_{\sigma=1}^{m} q_{\sigma}(n ; t) \Delta^{-1} r_{\sigma}(n ; t)$, where $q_{\sigma}(n ; t)$ and $r_{\sigma}(n ; t)$ are the eigenfunction and the adjoint eigenfunction of the dKP hierarchy respectively. In this section, we only consider one-component constrained dKP hierarchy, that is,

$$
L(n)_{-}=q(n ; t) \Delta^{-1} r(n ; t) .
$$

For the constrained dKP hierarchy (3.1), the conventional additional flow $\hat{\partial}_{m l}$ can not preserve the space of the Lax operators given by (3.1). Thus some modification must be needed, which is realized in [18] by adding the squared eigenfunction symmetry flow to $\hat{\partial}_{1, k}$, that is,

$$
\partial_{k}^{*} L=\left[-\left(M_{\Delta} L^{k}\right)_{-}+Y_{k-1}, L\right],
$$

Here

$$
Y_{k}=\sum_{j=0}^{k-1}\left(j-\frac{1}{2}(k-1)\right) L^{k-1-j}(q) \Delta^{-1}\left(L^{*}\right)^{j}(r), \quad k \geq 1,
$$

which spans the Virasoro algebra, i.e.

$$
\left[\partial_{l}^{*}, \partial_{k}^{*}\right]=(k-l) \partial_{k+l-1}^{*},
$$

on the space of the Lax operator. If let $\partial_{\alpha_{m}}$ be the squared eigenfunction symmetry generated by $\left(j-\frac{1}{2}(k-1)\right) L^{k-1-j}(q)$ and $\left(L^{*}\right)^{j}(r)$ for $j=1,2, \cdots, m$, then

$$
\partial_{k}^{*}=\hat{\partial}_{1, k-1}+\partial_{\alpha_{m}}
$$

Next we will investigate the action of $\partial_{k}^{*}$ on the tau function $\tau(n ; t)$.

In order to get the Virasoro action on the tau function of the constrained dKP hierarchy, we need to first rewrite (3.1) into the form of the tau function.

The negative part of arbitrary power of the the Lax operator for the constrained dKP hierarchy (3.1) has the following explicit form [18]

$$
\left(L^{k}\right)_{-}=\sum_{j=0}^{k-1} L^{k-j-1}(q) \Delta^{-1}\left(L^{*}\right)^{j}(r) .
$$

Before further investigating the constrained dKP hierarchy, the identity [14] below about the wave function and the adjoint wave function of the dKP hierarchy is needed.

$$
\frac{1}{\lambda} \hat{\Delta}_{z}\left(\psi_{B A}\left(n ; t+\left[\lambda^{-1}\right], \mu\right) \psi_{B A}^{*}(n ; t, \lambda)\right)=\frac{1}{z} \psi_{B A}(n ; t, \mu) \psi_{B A}^{*}\left(n ; t-\left[z^{-1}\right], \lambda\right) .
$$

where

$$
\hat{\Delta}_{z} f(t)=f\left(t-\left[z^{-1}\right]\right)-f(t) .
$$

Further by (2.15), (2.18) and (2.21), one can get

$$
\hat{\Delta}_{z} S(q(n ; t), r(n ; t))=-\frac{1}{z} q(n ; t) r\left(n-1 ; t-\left[z^{-1}\right]\right) .
$$


Next due to (2.7), (2.20) and (3.9),

$$
\begin{aligned}
& \lambda^{k} \psi_{B A}(n ; t, \lambda)=L(n)^{k}\left(\psi_{B A}(n ; t, \lambda)\right) \\
= & L(n)_{+}^{k}\left(\psi_{B A}(n ; t, \lambda)\right)+\sum_{j=0}^{k-1} L^{k-j-1}(q)(n ; t) S\left(\psi_{B A}(n ; t, \lambda),\left(L^{*}\right)^{j}(r)(n ; t)\right) \\
= & \partial_{t_{k}} \psi_{B A}(n ; t, \lambda)+\frac{1}{\lambda} \sum_{j=0}^{k-1} L^{k-j-1}(q)(n ; t)\left(L^{*}\right)^{j}(r)(n-1 ; t-[\lambda-1]) \psi_{B A}(n ; t, \lambda) \\
= & \partial_{t_{k}} \psi_{B A}(n ; t, \lambda)-\sum_{j=0}^{k-1} \psi_{B A}(n ; t, \lambda) \hat{\Delta}_{z} S\left(L^{k-j-1}(q),\left(L^{*}\right)^{j}(r)\right) .
\end{aligned}
$$

On the other hand, according to (2.11),

$$
\partial_{t_{k}} \ln \psi_{B A}(n ; t, \lambda)-\lambda^{k}=\hat{\Delta}_{z} \partial_{t_{k}} \ln \tau(n ; t) .
$$

Therefore the comparison of (3.10) and (3.11) leads to the following proposition.

Proposition 3.1. For the constrained dKP hierarchy (3.1), the tau function satisfies the following identities.

$$
\frac{\partial}{\partial t_{k}} \tau(n ; t)=\sum_{j=0}^{k-1} S\left(L^{k-1-j}(q),\left(L^{*}\right)^{j}(r)\right) \tau(n ; t) .
$$

Remark 1: Note that the right hand side of (3.12) is just the action of the squared eigenfunction symmetry on the tau function according to (2.23). Thus it is showed that the constrained dKP hierarchy can be derived just by identifying the squared eigenfunction symmetry with the negative of the time flow.

According to (2.23) and (2.26), the action of $\partial_{k}^{*}$ on tau function can be obtained.

Proposition 3.2. For the constrained $d K P$ hierarchy (3.1),

$$
\partial_{k}^{*} \tau(n ; t)=\frac{1}{2} \hat{W}_{k-1}^{(2)} \tau(n ; t)+\sum_{j=0}^{k-2}\left(\frac{1}{2}(k-2)-j\right) S\left(L^{k-2-j}(q),\left(L^{*}\right)^{j}(r)\right) \tau(n ; t),
$$

Next our goal is to express the second term in the right hand side of (3.13) as a differential operator acting on $\tau(n ; t)$ in the form similar to (2.27). For this, the following lemma must be needed. 
Lemma 3.1. For any eigenfunction $f(n ; t)$ and any adjoint eigenfunction $g(n ; t)$ of the constrained dKP hierarchy (3.1), the relation below holds

$$
\begin{aligned}
\frac{\partial}{\partial t_{k}} S(f, g)= & S\left(L^{k}(f), g\right)-S\left(f,\left(L^{*}\right)^{k}(g)\right) \\
& -\sum_{j=0}^{k-1} S\left(f,\left(L^{*}\right)^{j}(r)\right) S\left(L^{k-j-1}(q), g\right) .
\end{aligned}
$$

Proof. We first prove (3.14) holds for the wave function $\psi_{B A}(n ; t, \lambda)$ and the adjoint wave function $\psi_{B A}^{*}(n+1 ; t, \mu)$. Then by the spectral representation (2.15), it can be proved that it is true for $f$ and g.

From the proof of (3.10), one can know that

$$
\begin{aligned}
& \partial_{t_{k}} \Psi_{B A}(n ; t, \lambda)=\lambda^{k} \psi_{B A}(n ; t, \lambda) \\
& -\frac{1}{\lambda} \sum_{j=0}^{k-1} L^{k-j-1}(q)(n ; t)\left(L^{*}\right)^{j}(r)\left(n-1 ; t-\left[\lambda^{-1}\right]\right) \psi_{B A}(n ; t, \lambda) .
\end{aligned}
$$

Similarly, one can also obtain

$$
\begin{aligned}
& \partial_{t_{k}} \Psi_{B A}^{*}(n ; t, \mu)=-\mu^{k} \psi_{B A}^{*}(n ; t, \mu) \\
& +\frac{1}{\mu} \sum_{j=0}^{k-1} L^{k-j-1}(q)\left(n ; t+\left[\mu^{-1}\right]\right)\left(L^{*}\right)^{j}(r)(n-1 ; t) \psi_{B A}^{*}(n ; t, \mu) .
\end{aligned}
$$

Substituting (3.15) and (3.16) and according to (2.18), (2.20) and (3.9),

$$
\begin{aligned}
& \partial_{t_{k}} S\left(\psi_{B A}(n ; t, \lambda), \psi_{B A}^{*}(n+1 ; t, \lambda)\right)=\partial_{t_{k}}\left(\lambda^{-1} \psi_{B A}(n ; t, \lambda) \psi_{B A}^{*}\left(n ; t-\left[\lambda^{-1}\right], \mu\right)\right) \\
= & \lambda^{-1} \partial_{t_{k}} \psi_{B A}(n ; t, \lambda) \psi_{B A}^{*}\left(n ; t-\left[\lambda^{-1}\right], \mu\right)+\lambda^{-1} \psi_{B A}(n ; t, \lambda) \partial_{t_{k}} \psi_{B A}^{*}\left(n ; t-\left[\lambda^{-1}\right], \mu\right) \\
= & S\left(L(n)^{k}\left(\psi_{B A}(n ; t, \lambda)\right), \psi_{B A}^{*}(n+1 ; t, \mu)\right)-S\left(\psi_{B A}(n ; t, \lambda),\left(L(n)^{*}\right)^{k}\left(\psi_{B A}^{*}(n+1 ; t, \mu)\right)\right) \\
& +\sum_{j=0}^{k-1} S\left(\psi_{B A}(n ; t, \lambda),\left(L^{*}\right)^{j}(r)(n ; t)\right)\left(-\lambda^{-1} L^{k-j-1}(q)(n ; t) \psi_{B A}^{*}\left(n ; t-\left[\lambda^{-1}\right], \mu\right)\right. \\
& \left.-S\left(L^{k-j-1}(q)\left(n ; t-\left[\lambda^{-1}\right]\right), \psi_{B A}^{*}\left(n+1 ; t-\left[\lambda^{-1}\right], \mu\right)\right)\right) \\
= & S\left(L(n)^{k}\left(\psi_{B A}(n ; t, \lambda)\right), \psi_{B A}^{*}(n+1 ; t, \mu)\right)-S\left(\psi_{B A}(n ; t, \lambda),\left(L(n)^{*}\right)^{k}\left(\psi_{B A}^{*}(n+1 ; t, \mu)\right)\right) \\
& -\sum_{j=0}^{k-1} S\left(\psi_{B A}(n ; t, \lambda),\left(L^{*}\right)^{j}(r)(n ; t)\right) S\left(L^{k-j-1}(q)(n ; t), \psi_{B A}^{*}(n+1 ; t, \mu)\right) .
\end{aligned}
$$

So (3.14) holds for the (adjoint) wave function. And further by the spectral representation, it leads to the general case.

After the preparation above, now we can get the main result of this paper, which is showed in the proposition below.

Proposition 3.3. For the constrained dKP hierarchy (3.1),

$$
\partial_{k}^{*} \tau(n ; t)=\left(\sum_{i \geq 1}\left(i t_{i}+(-1)^{i-1} n\right) \frac{\partial}{\partial t_{i+k-1}}-\frac{k}{2} \frac{\partial}{\partial t_{k-1}}+\sum_{i=1}^{k-2} \frac{\partial^{2}}{\partial t_{i} \partial t_{k-1-i}}\right) \tau(n ; t) .
$$


Proof. By (3.13), it is only to show

$$
\sum_{j=0}^{k-2}\left(\frac{1}{2}(k-2)-j\right) S\left(L^{k-2-j}(q), L^{* j}(r)\right)=\frac{1}{2 \tau(n ; t)} \sum_{l=1}^{k-2} \frac{\partial^{2} \tau(n ; t)}{\partial t_{l} \partial t_{k-1-l}} .
$$

For this, according to (3.12),

$$
\begin{aligned}
\frac{\partial^{2} \tau(n ; t)}{\partial t_{l} \partial t_{k}}= & \sum_{i=0}^{k-1}\left(\frac{\partial}{\partial t_{l}} S\left(L^{k-1-i}(q),\left(L^{*}\right)^{i}(r)\right) \tau(n ; t)+S\left(L^{k-1-i}(q), L^{* i}(r)\right) \frac{\partial}{\partial t_{l}} \tau(n ; t)\right) \\
= & \sum_{i=0}^{k-1}\left(S\left(L^{k+l-1-i}(q),\left(L^{*}\right)^{i}(r)\right)-S\left(L^{k-1-i}(q),\left(L^{*}\right)^{i+l}(r)\right)\right) \tau(n ; t) \\
& +\sum_{i=0}^{k-1} \sum_{j=0}^{l-1}\left(S\left(L^{k-1-i}(q),\left(L^{*}\right)^{i}(r)\right) S\left(L^{l-1-j}(q),\left(L^{*}\right)^{j}(r)\right)\right. \\
& \left.-S\left(L^{k-1-i}(q),\left(L^{*}\right)^{j}(r)\right) S\left(L^{l-1-j}(q),\left(L^{*}\right)^{i}(r)\right)\right) \tau(n ; t) .
\end{aligned}
$$

Substitute (3.20) into the right hand side of (3.19),

$$
\begin{aligned}
& \frac{1}{\tau(n ; t)} \sum_{l=1}^{k-2} \frac{\partial^{2} \tau(n ; t)}{\partial t_{l} \partial t_{k-1-l}} \\
= & \sum_{l=1}^{k-2} \sum_{i=0}^{k-2-l}(\underbrace{S\left(L^{k-2-i}(q),\left(L^{*}\right)^{i}(r)\right)}_{(a)}-\underbrace{S\left(L^{k-2-i-l}(q),\left(L^{*}\right)^{i+l}(r)\right)}_{(b)}) \\
& +\sum_{l=1}^{k-2} \sum_{j=0}^{l-1} \sum_{i=0}^{k-2-l}(\underbrace{S\left(L^{k-2-i-l}(q),\left(L^{*}\right)^{i}(r)\right) S\left(L^{l-1-j}(q),\left(L^{*}\right)^{j}(r)\right)}_{(c)} \\
& -\underbrace{S\left(L^{k-2-i-l}(q),\left(L^{*}\right)^{j}(r)\right) S\left(L^{l-1-j}(q),\left(L^{*}\right)^{i}(r)\right)}_{(d)}) .
\end{aligned}
$$

Then

$$
\begin{aligned}
(a) & =\sum_{i=0}^{k-3} \sum_{i=l}^{k-2-i} S\left(L^{k-2-i}(q),\left(L^{*}\right)^{i}(r)\right)=\sum_{i=0}^{k-2}(k-2-i) S\left(L^{k-2-i}(q),\left(L^{*}\right)^{i}(r)\right), \\
(b) & =\sum_{l=1}^{k-2} \sum_{i=l}^{k-2} S\left(L^{k-2-i}(q),\left(L^{*}\right)^{i}(r)\right)=\sum_{i=1}^{k-2} \sum_{l=1}^{i} S\left(L^{k-2-i}(q),\left(L^{*}\right)^{i}(r)\right) \\
& =\sum_{i=0}^{k-2} i S\left(L^{k-2-i}(q),\left(L^{*}\right)^{i}(r)\right), \\
(c) & =\sum_{j=0}^{k-3} \sum_{i=0}^{k-j-3} \sum_{l=j+1}^{k-2-i} S\left(L^{k-2-i-l}(q),\left(L^{*}\right)^{i}(r)\right) S\left(L^{l-1-j}(q),\left(L^{*}\right)^{j}(r)\right) \\
& =\sum_{j=0}^{k-3} \sum_{i=0}^{k-j-3} \sum_{l=j+1}^{k-2-i} S\left(L^{k-2-i-l}(q),\left(L^{*}\right)^{j}(r)\right) S\left(L^{l-1-j}(q),\left(L^{*}\right)^{i}(r)\right)=(d),
\end{aligned}
$$


where in the first identity of $(b)$-term, $i$ becomes into $i-l$, and for the second identity of $(c)$-term, we have let $l \rightarrow k-1-i+j-l$. Therefore after substituting $(a)(b)(c)$ into $\frac{1}{\tau(n ; t)} \sum_{l=1}^{k-2} \frac{\partial^{2} \tau(n ; t)}{\partial t_{l} \partial t_{k-1-l}}$, (3.19) is proved.

Remark 2: For the constrained dKP hierarchy with $m$ components $(m>1)$, the relations (3.12), (3.13) and (3.14) still hold. But (3.19) will become into

$$
\begin{aligned}
\frac{\partial^{2} \tau(n ; t)}{\partial t_{l} \partial t_{k}}= & \sum_{\sigma=1}^{m} \sum_{i=0}^{k-1} S\left(L^{k+l-1-i}\left(q_{\sigma}\right),\left(L^{*}\right)^{i}\left(r_{\sigma}\right)\right)-S\left(L^{k-1-i}\left(q_{\sigma}\right),\left(L^{*}\right)^{i+l}\left(r_{\sigma}\right)\right) \\
& +\sum_{\sigma, \rho=1}^{m} \sum_{i=0}^{k-1} \sum_{j=0}^{l-1}\left(S\left(L^{k-1-i}\left(q_{\sigma}\right),\left(L^{*}\right)^{i}\left(r_{\sigma}\right)\right) S\left(L^{l-1-j}\left(q_{\rho}\right),\left(L^{*}\right)^{j}\left(r_{\rho}\right)\right)\right. \\
& \left.-S\left(L^{k-1-i}\left(q_{\sigma}\right),\left(L^{*}\right)^{j}\left(r_{\rho}\right)\right) S\left(L^{l-1-j}\left(q_{\rho}\right),\left(L^{*}\right)^{i}\left(r_{\sigma}\right)\right)\right)
\end{aligned}
$$

Though the corresponding $(a)(b)$-terms in (3.21) can lead to the corresponding term in the left hand side of (3.19), the $(c)(d)$-terms can not be cancelled.

Remark 3: $(-1)^{i-1} n$ terms in (3.18) is the difference between the dKP hierarchy and the usual KP hierarchy. And by (3.18), one can easily show $\left[\partial_{k}^{*}, \partial_{l}^{*}\right]=(k-l) \partial_{k+l-1}^{*}$ on the space of the tau functions.

\section{Conclusions and Discussions}

In this paper, the action of the Virasoro symmetry on the tau function of the constrained dKP hierarchy is derived (see Proposition 4), which is expected to be helpful in the study of the algebraic constraint of the discrete system and the links between the dKP hierarchy and the matrix models. Note that if letting $t_{i}+(-1)^{i-1} n / i \rightarrow t_{i}$, then (3.18) will be the same as the case of the usual KP hierarchy, which is consistent with the relation (2.13) between $\tau(t)$ of the usual KP hierarchy and $\tau(n ; t)$ of the dKP hierarchy. What's more, the constraint on the tau function of the constrained dKP hierarchy is showed in (3.12) and an important property about the squared eigenfunction potential of the constrained dKP hierarchy is obtained in (3.14).

\section{Acknowledgments}

This work is supported by the NSFC (Grant Nos. 11226196, 11301526 and 11371361) and the Fundamental Research Funds for the Central Universities (Grant No. 2012QNA45).

\section{References}

[1] M. Adler, T. Shiota and P. van Moerbeke, A Lax representation for the vertex operator and the central extension, Comm. Math. Phys. 171 (1995) 547-588.

[2] H. Aratyn, E. Nissimov and S. Pacheva, Constrained KP hierarchies: additional symmetries, DarbouxBäcklund solutions and relations to multi-matrix models, Int. J. Mod. Phys. A 12 (1997) 1265-1340.

[3] H. Aratyn, E. Nissimov and S. Pacheva, Method of squared eigenfunction potentials in integrable hierarchies of KP type, Comm. Math. Phys. 193 (1998) 493-525.

[4] H. H. Chen, Y. C. Lee and J. E. Lin, On a new hierarchy of symmetry for the Kadomtsev-Petviashvili equation, Physica D 9 (1983) 439-445.

[5] J. P. Cheng, K. L. Tian and J. S. He, The additional symmetries for the BTL and CTL hierarchies, J. Math. Phys. 52 (2011) 053515. 
[6] J. P. Cheng, Y. Tian, Z. W. Yan and J. S. He, The generalized additional symmetries of the two-Toda lattice hierarchy, J. Math. Phys. 54 (2013) 023513.

[7] L. A. Dickey, On additional symmetries of the KP hierarchy and Sato's Bäcklund transformation, Comm. Math. Phys. 167 (1995) 227-233.

[8] L. A. Dickey, Soliton equations and Hamiltonian systems (2nd Edition) (World scientific, Singapore, 2003).

[9] A. S. Fokas and B. Fuchssteiner, The hierarchy of the Benjamin-Ono equation, Phys. Lett. A 86 (1981) 341-345.

[10] W. Fu, L. Huang, K. M. Tamizhmani and D. J. Zhang, Integrable properties of the differential-difference Kadomtsev-Petviashvili hierarchy and continuum limits, arXiv:1211.3585.

[11] L. Haine and P. Iliev, Commutative rings of difference operators and an Adelic flag manifold, Int. Math. Res. Not. 6 (2000) 281-323.

[12] J. S. He, K. L. Tian, A. Foerster and W. X. Ma, Additional symmetries and string equation of the CKP hierarchy, Lett. Math. Phys. 81 (2007) 119-134.

[13] B. A. Kupershimidt, Discrete Lax equations and difference calculus, Asterisque 123 (1985) 1-212.

[14] C. Z. Li, J. P. Cheng, K. L. Tian, M. H. Li and J. S. He, Ghost symmetry of the discrete KP hierarchy, arXiv:1201.4419.

[15] C. Z. Li and J. S. He, Dispersionless bigraded Toda hierarchy and its additional symmetry, Rev. Math. Phys. 24 (2012) 1230003.

[16] C. Z. Li, J. S. He and Y. C. Su, Block type symmetry of bigraded Toda hierarchy, J. Math. Phys. 53 (2012) 013517.

[17] M. H. Li, J. P. Cheng and J. S. He, The gauge transformation of the constrained semi-discrete KP hierarchy, Mod. Phys. Lett. B 27 (2013) 1350043.

[18] M. H. Li, C. Z. Li, K. L. Tian, J. S. He and Y. Cheng, Virasoro type algebraic structure hidden in the constrained discrete KP hierarchy, J. Math. Phys. 54 (2013) 043512.

[19] S. W. Liu, Y. Cheng and J. S. He, The determinant representation of the gauge transformation for the discrete KP hierarchy, Sci. China Math. 53 (2010) 1195-1206.

[20] S. W. Liu and Y. Cheng, Sato Backlund transformation, additional symmetries and ASvM formula for the discrete KP hierarchy, J. Phys A: Math. Theor. 43 (2010) 135202.

[21] A. Yu. Orlov and E. I. Schulman, Additional symmetries for integrable systems and conformal algebra repesentation, Lett. Math. Phys. 12 (1986) 171-179.

[22] Y. Q. Yao, X. J. Liu and Y. B. Zeng, A new extended discrete KP hierarchy and a generalized dressing method, J. Phys. A: Math. Theor. 42 (2009) 454026.

[23] X. L. Sun, D. J. Zhang, X. Y. Zhu and D. Y. Chen, Symmetries and Lie algebra of the differentialdifference Kadomstev-Petviashvili hierarchy, Mod. Phys. Lett. B 24 (2010) 1033-1042.

[24] H. F. Shen and M. H. Tu, On the constrained B-type Kadomtsev-Petviashvili hierarchy: Hirota bilinear equations and Virasoro symmetry, J. Math. Phys. 52 (2011) 032704.

[25] K. L. Tian, J. S. He, J. P. Cheng and Y. Cheng, Additional symmetries of constrained CKP and BKP hierarchies, Sci. China Math. 54 (2011) 257-268.

[26] M. H. Tu, On the BKP hierarchy: Additional symmetries, Fay identity and Adler-Shiota- van Moerbeke formula. Lett. Math. Phys. 81 (2007) 91-105.

[27] P. van Moerbeke, Integrable fundations of string theory, in Lectures on Integrable systems, eds. O. Babelon, P. Cartier, Y. Kosmann-Schwarzbach (World Scientific, Singapore, 1994) pp. 163-267. 